\title{
Holographic memory with localized recording
}

\author{
Christophe Moser and Demetri Psaltis
}

\begin{abstract}
We experimentally demonstrate and characterize a memory module that features selective page erasure and readout persistence using the localized recording method in doubly doped $\mathrm{LiNbO}_{3}$. Pages of information can be selectively erased without partially erasing the whole memory. Data pages can be written over erased pages multiple times. Information is read millions of times before refreshing is required. We quantify the optical quality of the holograms by measuring their signal-to-noise ratio for a memory size up to 100 holograms. A compact phase-conjugate readout architecture is also presented and experimentally demonstrated. (C) 2001 Optical Society of America
\end{abstract}

OCIS codes: $210.0210,210.2860$.

\section{Introduction}

We present a read-write memory with a selective erase and rewrite capability based on the localized recording method in doubly doped $\mathrm{LiNbO}_{3}: \mathrm{Fe}, \mathrm{Mn}$ crystals. ${ }^{1}$ Figure 1 shows the localization idea. Recording in these doubly doped crystals requires the simultaneous presence of the recording beam and a sensitizing beam. The sensitizing beam defines the area to be recorded. In Fig. 1 the sensitizing and reference recording beams are focused by a cylindrical lens. Within the depth of focus, the sensitizing light creates a thin plane with a thickness of a few tens of micrometers in the crystal. Multiplexing is then performed when the reference and sensitizing beams are moved to an adjacent location. Selective erasure is performed when the desired hologram's spatial location is illuminated only with the sensitizing beam. In Ref. 2 we demonstrated the feasibility of this scheme by recording multiple localized holograms. This holographic technique can be seen as analogous to a layered three-dimensional optical storage system. ${ }^{3,4}$

In this paper we report on the experimental measurement of the signal-to-noise ratio (SNR) as a function of the number of localized holograms and their

When this research was performed the authors were with the Department of Electrical Engineering, California Institute of Technology, 1201 East California Boulevard, Mail Stop 136-93, Pasadena, California 91125. C. Moser (e-mail: moser@ondax.com) is now with Ondax, 850 East Duarte, Monrovia, California 91016.

Received 20 October 2000; revised manuscript received 20 March 2001.

0003-6935/01/03909-06\$15.00/0

(C) 2001 Optical Society of America lateral extent (thickness of the focused plane). We also investigate the SNR after multiple selective refresh-erasure cycles of a localized hologram at the same location. A typical recording erasing curve, by use of 90-deg recording geometry, with two recording plane waves [wavelength is $532 \mathrm{~nm}$, beam diameter is $2 \mathrm{~mm}$, ordinary polarization, intensity is $80(\mathrm{~mW} /$ $\mathrm{cm}^{2}$ )/beam, intensity of the violet beam is $20 \mathrm{~mW} /$ $\mathrm{cm}^{2}$, and wavelength is $401 \mathrm{~nm}$ ] is shown in Fig. 2 . The recording is done with the violet beam on. During readout, the violet beam is turned off, and the reference beam with twice the intensity reads out the hologram in the Bragg condition. The erasing curve is fitted with a biexponential function giving two time constants, $\tau_{e 1}=1083 \mathrm{~s}$ and $\tau_{e 2}=30403 \mathrm{~s}$ corresponding, respectively, to the fast erasure of the grating in $\mathrm{Fe}$ and a slower erasure of the grating in $\mathrm{Mn} .{ }^{5}$ The crystal was oxidized for $6 \mathrm{~h}$ at $900{ }^{\circ} \mathrm{C}$ in an $\mathrm{O}_{2}$ atmosphere. The oxidation state could not be reduced further by longer oxidation. The slow erasure time constant $\tau_{e 2}$ is approximately 15 times shorter than that obtained in a similar crystal with half of the concentration of $\mathrm{Fe}_{2} \mathrm{O}_{3}\left(0.075\right.$-wt. $\% \mathrm{Fe}_{2} \mathrm{O}_{3}$, transmission geometry). ${ }^{6}$ The ratio of the erasing time constant to the recording time constant is equal to $\tau_{e 2} /$ $\tau_{w}=140$.

In the experiments that are presented next, pages of information are encoded by a binary spatial light modulator (SLM). The reference and sensitizing beams are copropagating and focused in the crystal according to Fig. 3. The localized holograms are recorded with equal power in the reference and signal beams to maximize the recording slope, thereby minimizing the recording time of each hologram. ${ }^{2} \mathrm{We}$ observed that when holograms are not recorded to saturation, as is the case in the recording of Fig. 2, the 


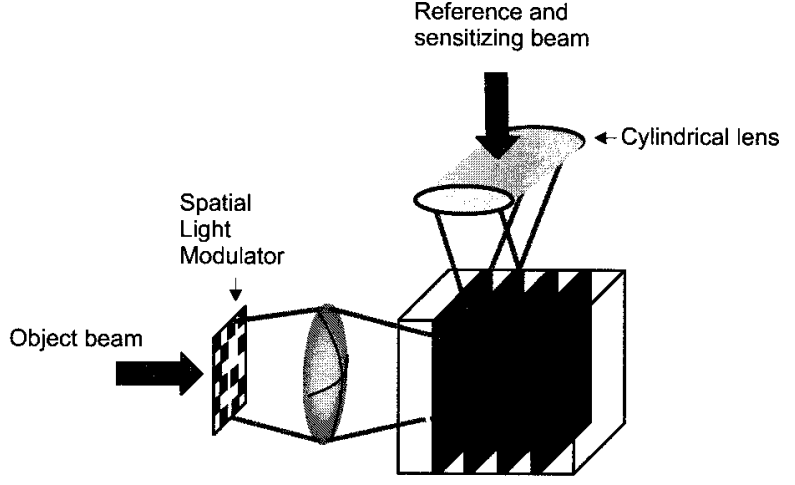

Fig. 1. Localized recording in doubly doped $\mathrm{LiNbO}_{3}$.

persistence on readout of the holograms varies with the recording time. The persistence parameter $\beta^{2}$ is defined as the ratio between the diffraction efficiency after sufficient readout (flat part of the erasing curve) and the diffraction efficiency immediately after recording. We experimentally measured the persistence as a function of recording time. Holograms are recorded by the setup shown in Fig. 3 with a focused spot size of $35 \mu \mathrm{m}$. Figure 4(a) shows recording and erasing curves for increasing recording times. The persistence $\beta^{2}$ is extracted from the recording curves of Fig. 4(a), and the result is presented in Fig. 4(b). We observe that holograms recorded for short times have poor persistence. This result is independent of the amount of presensitizing time with the violet beam. We attribute this result to the dynamics of the recording. Bleaching and sensitizing in $\mathrm{LiNbO}_{3}: \mathrm{Fe}, \mathrm{Mn}$ are typically slow mechanisms. After the crystal is presensitized for some time, the iron traps are filled up with electrons. During the early recording period, the grating is built mainly with electrons coming from the filled-up iron centers. After sufficient recording time, equilibrium is

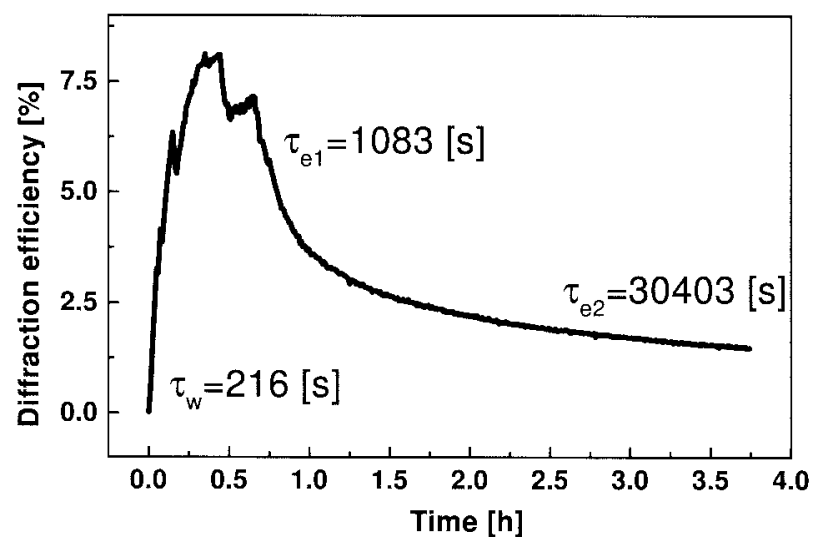

Fig. 2. Recording and readout curve for a plane-wave hologram in a 90-deg-cut $\mathrm{LiNbO}_{3}$ crystal doped with 0.15 -wt. $\% \mathrm{Fe}_{2} \mathrm{O}_{3}$ and 0.015-wt. \% MnO. The recording beams are from a solid-state green laser (wavelength $532 \mathrm{~nm}$, ordinary polarization, $1 / e^{2}$ beam diameter $2.0 \mathrm{~mm}$, and intensity $80\left(\mathrm{~mW} / \mathrm{cm}^{2} /\right.$ beam). The sensitizing light is from a laser diode (Nichia, wavelength $401 \mathrm{~nm}$, linearly polarized, intensity $20 \mathrm{~mW} / \mathrm{cm}^{2}$ ).

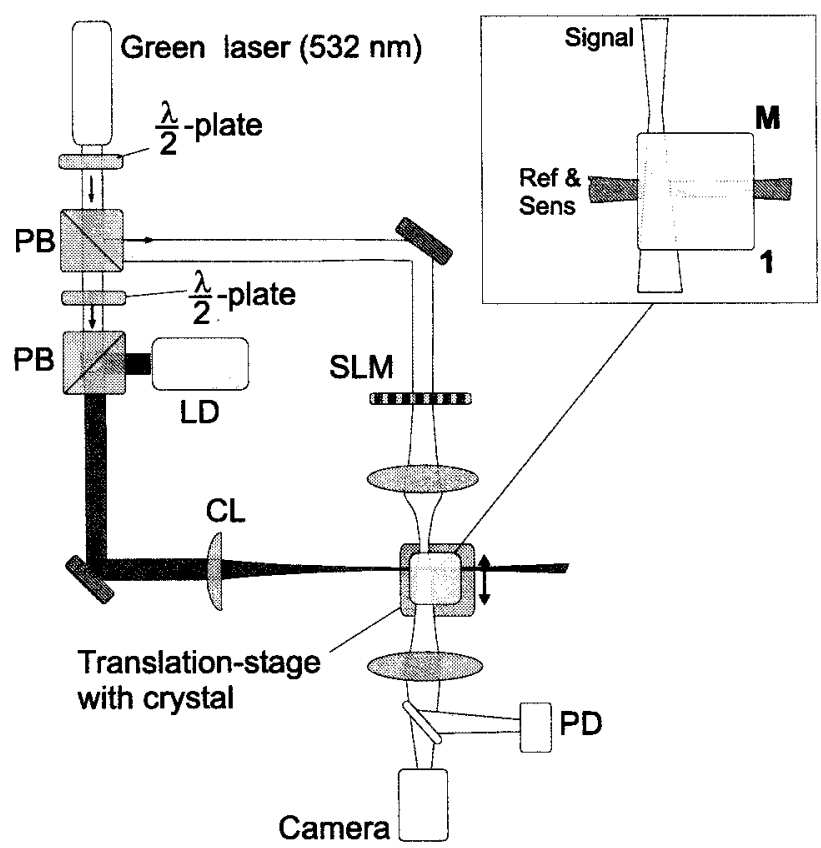

Fig. 3. Recording setup for the SNR measurement. LD, laser diode; CL, cylindrical lens; PD, photodiode; PB, polarizing beam splitter.

reached between the rate of electrons transferred from $\mathrm{Mn}$ to $\mathrm{Fe}$ and vice versa. The grating is then efficiently recorded in the Mn centers, and persistence reaches its stable value as shown in Fig. 4(b). For recording in the green at $532 \mathrm{~nm}$, the maximum persistence obtained is $\beta^{2}=0.34$, meaning that ap-
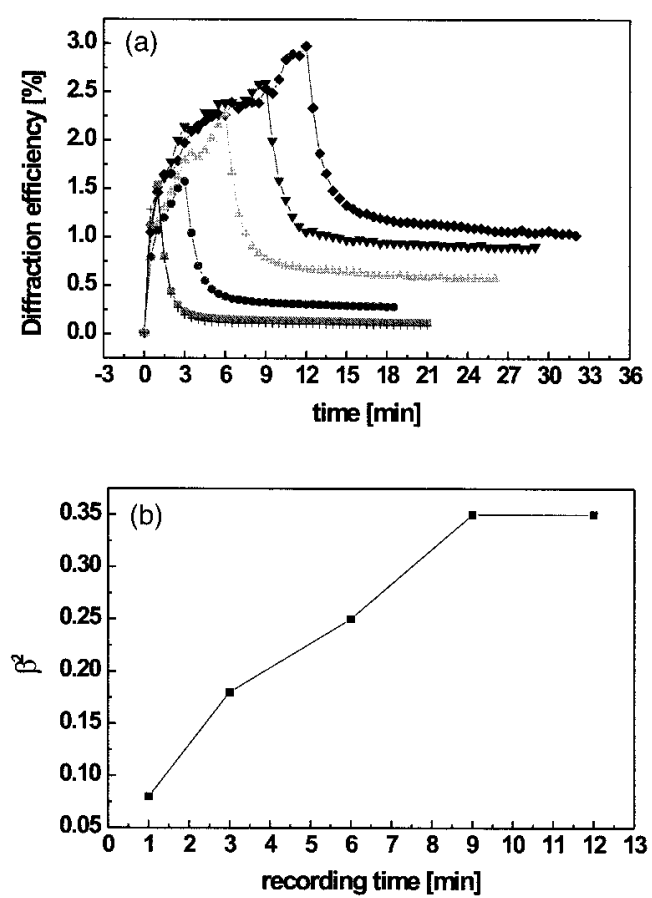

Fig. 4. (a) Recording curves with a focused green 532-nm reference at $35 \mu \mathrm{m}\left(1 / e^{2}\right.$ value) and erasing with reference beam only. (b) Persistence $\beta^{2}$ as a function of recording time. 
proximately a third of the diffraction efficiency remains after initial erasure. When the memory is recorded initially with the localized method, it is important to record the holograms for an amount of time long enough to achieve maximum persistence. The final diffraction efficiency of the holograms after the memory is written will then be maximized.

\section{Signal-to-Noise Ratio of Localized Holograms}

We present the SNR of reconstructed holograms for two experiments. In the first experiment, a focused reference beam with a $1 / e^{2}$ lateral spot size equal to $100 \mu \mathrm{m}$ is used. In the second experiment, the focused reference beam has a lateral spot size equal to $25 \mu \mathrm{m}$. The spot size is measured with a knife edge translated orthogonally to the beam propagation direction. The lateral spot size $W(z)$ in the crystal increases with distance $z$ as $W(z)=2 W_{o}\left(1+z / z_{o}\right)^{1 / 2}$, where $z_{o}=\pi W_{o}{ }^{2} n / \lambda_{o}$ is the depth of focus, $W_{o}$ is the optimal waist at focus, and $n$ is the index of refraction for ordinary polarization.

The setup for the SNR measurement is shown in Fig. 3. The reference beam is focused by a cylindrical lens of focal length $4 \mathrm{~cm}$. The sensitizing beam from a laser diode (Nichia, $401 \mathrm{~nm}$ ) is copropagating with the green (Coherent, $532 \mathrm{~nm}$, ordinary polarization) reference beam. A SLM of $640 \times 480$ pixels (30 $\mu \mathrm{m}$ in size) is used to display random binary patterns. The 4- $f$ imaging system is not pixel matched. Instead $4 \times 4 \mathrm{SLM}$ pixels are used to represent one data pixel. The imaging system from the SLM to the detector array consists of an achromat lens of focal length $55 \mathrm{~mm}$ placed in front of the SLM and an objective Nikon lens with a numerical aperture of 0.7 . The imaging system's magnification of 0.29 allows us to image the entire SLM surface onto the camera. A removable mirror can be placed after the second imaging lens to measure the diffracted power while the camera is left in place. The focal plane of the first lens is located $3 \mathrm{~mm}$ before the crystal's surface; therefore the holograms are recorded in the Fresnel region. A 0.8-mm-diameter iris is placed at the focal plane of the first imaging lens to filter out the high orders generated by the SLM. The signal beam propagates through the crystal close to one of its edges. This ensures that the violet light sensitizes efficiently the entire area to be recorded as shown in the inset of Fig. 3. The holograms are recorded from position 1 (exit crystal facet) to $\mathrm{M}$ (entrance crystal facet). In this configuration, the signal beam interferes with the reference beam before it propagates through the recorded holograms, thus reducing distortion of the signal. At the overlap between the focused reference and the signal beams, the signal diameter is $1.5 \mathrm{~mm}$. The waist of the focused beam coincides with the entrance facet of the crystal. The spot size of the focused beam increases by $2.2 \%$ and $30 \%$ after a propagation distance of $1.5 \mathrm{~mm}$ for the $100-$ and $25-\mu \mathrm{m}$ spot sizes, respectively.

The experiments are performed with a congruent 90-deg-cut $\mathrm{LiNbO}_{3}$ crystal doped with 0.15 -wt. \% $\mathrm{Fe}_{2} \mathrm{O}_{3}$ and 0.015-wt. \% $\mathrm{MnO}$. The crystal dimen-
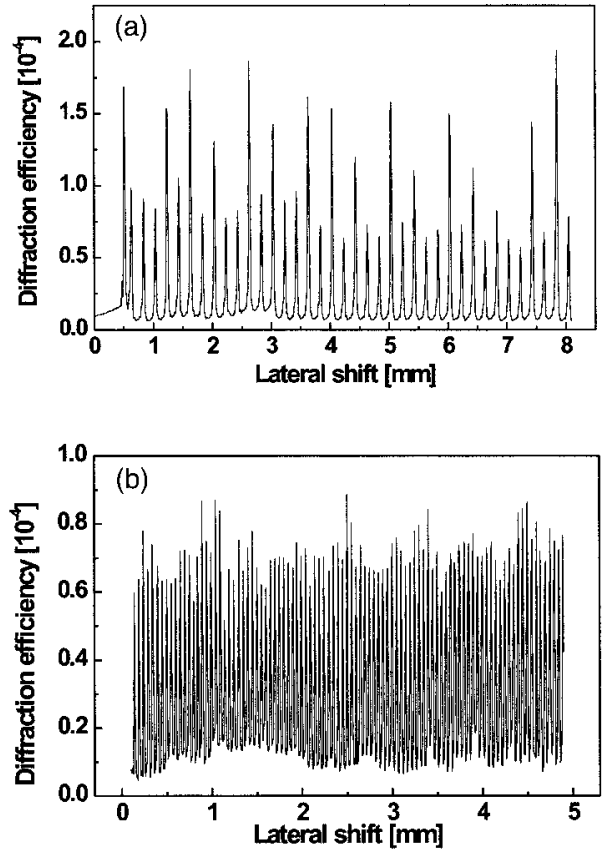

Fig. 5. (a) Spatial multiplexing of 40 localized holograms. (b) Spatial multiplexing of 100 localized holograms.

sions are $1 \mathrm{~cm} \times 1 \mathrm{~cm} \times 1 \mathrm{~cm}$. The signal, reference, and violet beam intensities are 30,300 , and $100 \mathrm{~mW}$ / $\mathrm{cm}^{2}$, respectively, for the $100-\mu \mathrm{m}$ spot size experiment. For the $25-\mu \mathrm{m}$ spot size, the intensities are 60,200 , and $600 \mathrm{~mW} / \mathrm{cm}^{2}$, respectively. A diffraction efficiency of $5 \times 10^{-5}$ is the smallest value that gives a good contrast image on a video camera sampling at $30 \mathrm{~Hz}$, from which we compute the SNR. The spacing between each localized hologram is chosen to be twice the lateral spot size to avoid erasure of the previous hologram while we are sensitizing the next hologram. Each hologram is sensitized for 5 min and recorded for 3 min with a different random binary bit pattern displayed on the SLM. No exposure schedule is needed to write the entire memory. Figures 5(a) and 5(b) show the diffraction efficiency versus position for the first and second experiments, respectively. Each peak corresponds to the diffraction efficiency of a hologram stored at a different spatial location as the crystal is translated in the direction perpendicular to the reference beam. Samples of reconstructed holograms from both experiments are shown in Figs. 6(a) and 6(b).

To measure the SNR, we use five windows of $30 \times$ 30 superpixels (one superpixel is composed of $4 \times 4$ camera pixels) located on the four corners and at the center of the image. For each superpixel, the edges are left out, so $3 \times 3$ pixels effectively form a superpixel. The SNR is computed by the formula

$$
\mathrm{SNR}=\frac{\mu_{1}-\mu_{0}}{\left(\sigma_{1}^{2}+\sigma_{0}^{2}\right)}
$$

where $\mu_{i}$ and $\sigma_{i}(i=1,0)$ are the mean and variance, respectively, of the fitted Gaussian distribution of the 

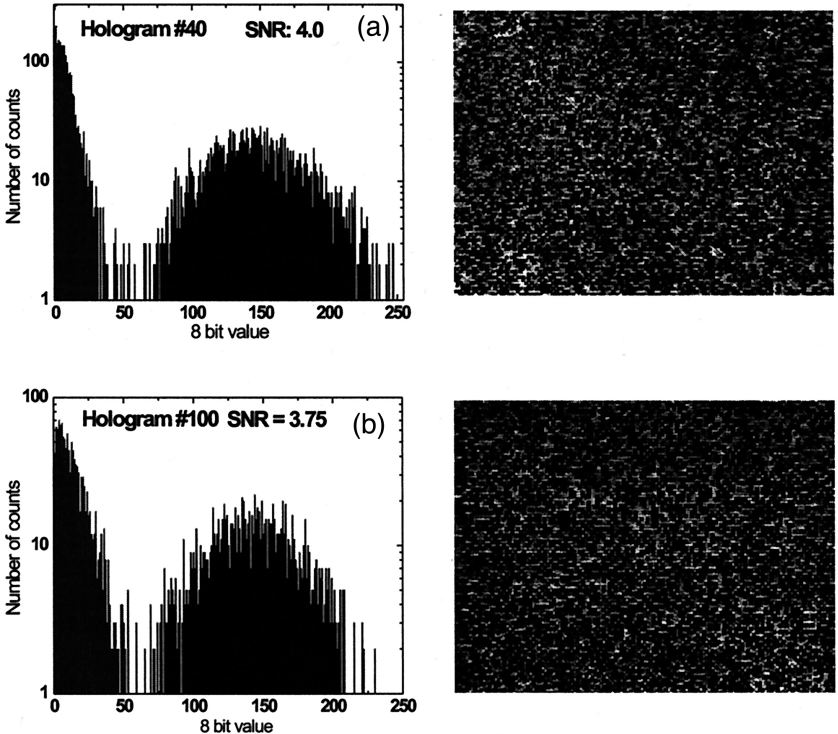

Fig. 6. (a) Sample reconstructions and SNR values from the experiment with 40 holograms. (b) Sample reconstructions and SNR values from the experiment with 100 holograms.

ones and zeros. The SNR is computed for holograms located in the front, middle, and rear of the crystal.

The average SNR value is 4.2 for the experiment with a $100-\mu \mathrm{m}$ lateral spot size and 3.7 for the experiment with a $25-\mu \mathrm{m}$ lateral spot size. In both experiments, the SNR is uniform across the crystal. We attribute the loss in SNR of 0.5 when the lateral spot size decreases from 100 to $25 \mu \mathrm{m}$ to the longer exposure of the crystal illuminated by the signal beam during the second experiment of 100 holograms. The remnant electrons in Fe act as absorption centers for the green (532-nm) light and causes distortion of the signal because of the photovoltaic effect. Imag-

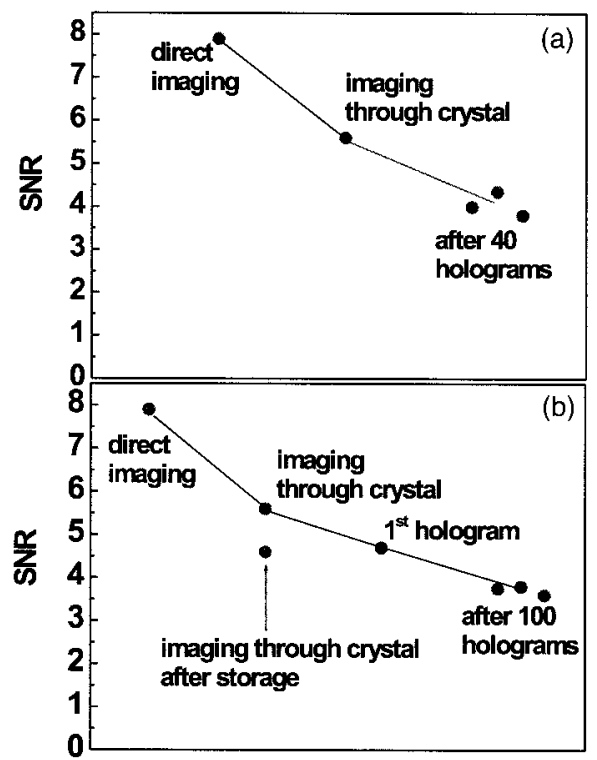

Fig. 7. SNR comparison with a focused reference beam of spot size (a) $100 \mu \mathrm{m}$ and (b) $25 \mu \mathrm{m}$.

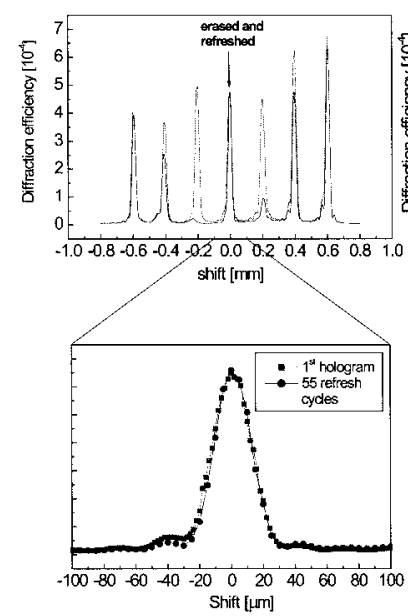

(a)

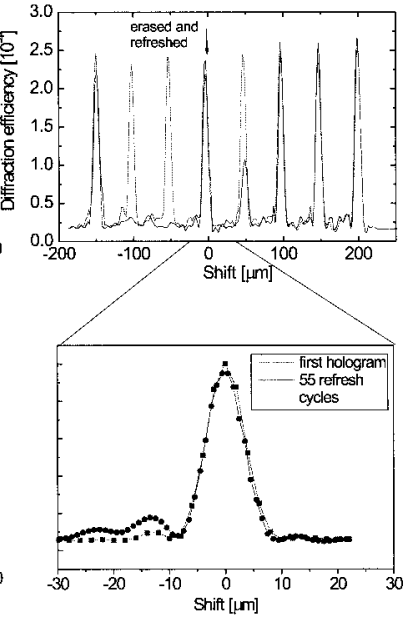

(b)
Fig. 8. Diffraction efficiency versus position before and after 55 refresh cycles of the central hologram: (a) $100-\mu \mathrm{m}$ reference focus, (b) $25-\mu \mathrm{m}$ reference focus.

ing the SLM through the crystal after the storage of 100 holograms shows a degradation of the SNR by one compared with the SNR measured before the full storage. Figure 7 summarizes the SNR measurements for both experiments. These results show that the SNR of a single hologram does not decay when the spot size of the reference beam is reduced, which suggests that an even smaller focus could be used; however, fanning of the signal beam becomes the limiting factor when long exposures are needed.

\section{Selective Erasure}

Localized recording allows selective erasure of a particular page of data. In this section we investigate the effect of multiple erasure-refresh cycles on the SNR for the two reference beam spot sizes (100 and $25 \mu \mathrm{m})$.

Seven holograms are recorded with a reference beam lateral spot size of $100 \mu \mathrm{m}$. The center hologram is then erased and refreshed 55 times. The green reference and violet beams are used together to erase the hologram. The hologram is refreshed for 3 min and erased for $10 \mathrm{~min}$. The beam intensities are the same as in the previous experiments. The experiment lasted $12 \mathrm{~h}$. Figure 8(a) shows a plot of the diffraction efficiency versus the spatial position before and after the 55 erase-refresh cycles. The bottom plot is a finer measurement of the diffraction efficiency versus position. It illustrates that there is no broadening of the selectivity curve because of the erase-refresh cycles. The immediate adjacent holograms are almost totally erased after the process. This is due to fanning and scattering from the surfaces of the crystal by the green reference and sensitizing beams. The SNRs of the recycled holograms before and after the 55 cycles are identical and equal to 4.5 as shown in Fig. 9(a).

An identical experiment is performed with a smaller lateral spot size of the reference beam of 25 

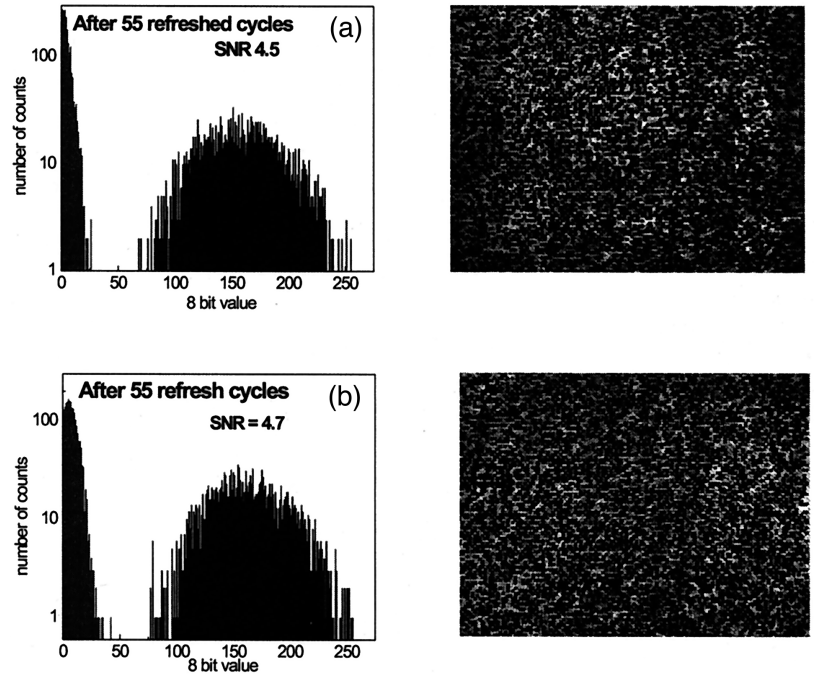

Fig. 9. Histogram and reconstruction of the central hologram before and after the refresh cycle: (a) $100-\mu \mathrm{m}$ focus, (b) $25-\mu \mathrm{m}$ focus.

$\mu \mathrm{m}$. After 55 erase-refresh cycles, Fig. 8(b) shows that the small focused reference beam does not influence the selectivity of the central hologram. We observe an erasure asymmetry of the adjacent holograms caused by ultraviolet erasure. The two adjacent holograms located at the left of the central hologram are erased totally whereas the adjacent hologram on the right is erased by half of its original value. This is due to the preferential fanning direction to one side. When the reference spot size is reduced, the intensity of the green recording beam and sensitizing beam increases, enhancing the effect of fanning which causes erasure of farther neighboring holograms. The SNRs before and after the erasing-refreshing cycles are identical and equal to 4.7 as shown in Fig. 9(b).

These SNR values are similar to those obtained with distributed volume recording. It demonstrates that localized recording is a promising approach toward a practical selective read-write optical memory. The erasure of neighboring holograms caused by fanning can be reduced when a superluminescent diode with a low temporal coherence length is used. A limitation of the experiment shown above is the memory capacity. To increase the capacity, a longer crystal could be used, but in practice, the crystal length is limited because the signal beam needs to be coupled in the crystal without bouncing off the walls. We present the following implementation, based on phase conjugation, that allows the memory capacity to grow linearly with the crystal length.

\section{Compact Phase-Conjugate Module}

Figure 10 illustrates the geometry. In this architecture, the reference beam focus spot size can be small, and we keep the signal beam diameter constant throughout the crystal by bouncing it off the crystal walls. The signal beam picks up information from the SLM and is focused into the crystal, positioned

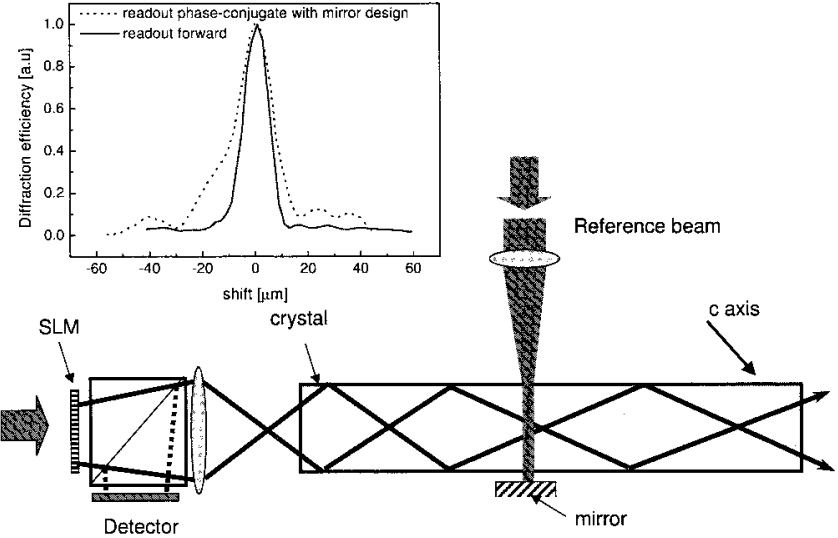

Fig. 10. Compact module by use of a mirror for phase conjugation. Also shown is the spatial selectivity of the forward and conjugate reconstruction.

close to the Fourier plane of the lens. The crystal slab acts as a waveguide for the signal beam. The signal lateral dimension is then limited by the crystal thickness. Forward reconstruction would yield a distorted image because of the waveguiding effect. When the conjugate reference beam illuminates the hologram, the waveguiding effect is effectively undone, and the image can be recovered by the beam splitter placed in front of the crystal. Others have used crystal fibers to store and retrieve holograms using the phase conjugate. ${ }^{7,8}$ The focused reference beam spot size is equal to $25 \mu \mathrm{m}$. To test the concept, we use a crystal slab of $\mathrm{LiNbO}_{3}$ doped with 0.1-wt. $\% \mathrm{Fe}_{2} \mathrm{O}_{3}$ with a thickness of $1 \mathrm{~mm}$ and a length of $20 \mathrm{~mm}$ (along the signal beam direction) by $10 \mathrm{~mm}$. The $c$ axis of the crystal is oriented as shown in Fig. 10. A mirror is positioned at the focal plane of the cylindrical lens. The crystal is positioned between the cylindrical lens and the mirror ( 5 $\mathrm{mm}$ from the mirror). The reference beam is incident on the crystal near the middle of the crystal. The signal beam is focused by a cylindrical lens of focal length $f=7.5 \mathrm{~cm}$ into the crystal. The SLM is implemented by a mask of a random bit pattern of pixel size $80 \mu \mathrm{m} \times 80 \mu \mathrm{m}$. The orientation of the crystal's $c$ axis is such that the hologram is recorded efficiently by the interference of the signal beam and the reference beam reflected from the mirror. During readout, the forward reference beam is used as the phase-conjugate readout beam while a shutter (cardboard) is placed between the crystal and the mirror.

We measure the spatial selectivity of the hologram using the forward and conjugate reconstruction by translating the crystal in a direction orthogonal to the forward and conjugate beams. The result is shown in Fig. 10. The forward reconstruction shows a clean selectivity curve with $20-\mu \mathrm{m}$ selectivity. We observe a broadening of the selectivity curve for the phase-conjugate readout that is attributed to the distortion of the reference beam focused through the crystal. The SNR of the reconstruction is measured 


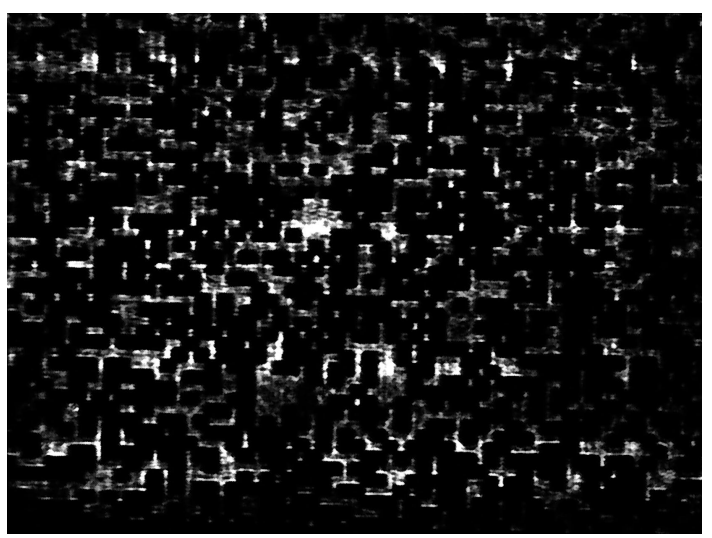

Fig. 11. Reconstructed hologram by phase conjugation.

as explained above, and Fig. 11 shows the result. The SNR of the conjugate reconstruction is equal to 4 and demonstrates the practicality of the reconstruction by phase conjugation when a small focused beam is used in a crystal slab.

\section{Memory Capacity}

Using the phase-conjugate implementation, and the architecture of Fig. 12, we can compute the memory capacity as follows. We assume that the crystal has a transverse dimension $H$ by $D$. The pixel size is denoted by $b$. The SLM has $N_{H} \times N_{D}$ pixels and dimension $H \times D$. The SLM is placed in front of the beam splitter, and the detector array is placed below the beam splitter to grab the phase-conjugate reconstruction. The number of data pages that can be recorded in a crystal of length $L$ is equal to

$$
N=\frac{L}{2 W(H)},
$$

where $W(H)$ is the waist of the focused reference beam after propagating a distance $H$. Given the crystal thickness $H$, the optimal waist $W_{o}$ at the entrance face that minimizes diffraction is equal to $(\lambda H / \pi n)^{1 / 2}$, where $n$ is the index of refraction of the crystal. $W(H)$ is then equal to $2 W_{o}$.

The crystal thickness $H$ is equal to the SLM verti-

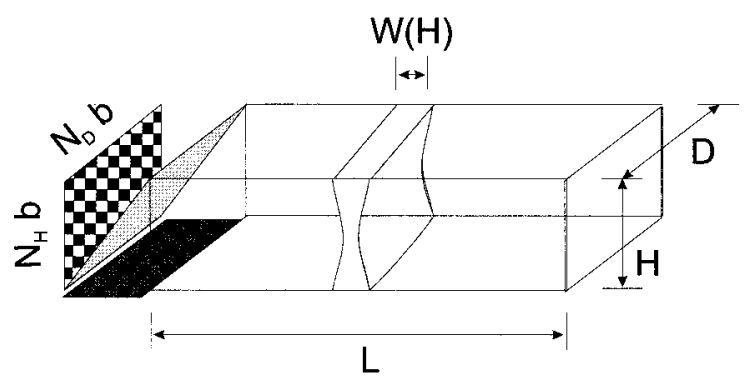

Fig. 12. Memory capacity for the phase-conjugate module. cal size $N_{H} b$, where $b$ is the pixel size. Eq. (2) is then equal to

$$
N=\frac{L \sqrt{n \pi}}{4 \sqrt{\lambda N_{H} b}} .
$$

Choosing the pixel size $b=2 \mu \mathrm{m}$ and crystal dimensions $H=1 \mathrm{~mm}, D=20 \mathrm{~mm}, L=50 \mathrm{~mm}$, we obtain $N_{H}=500$ and $N_{D}=10,000$. We obtain capacity by multiplying the result of Eq. (3) by the number of pixels per page $\left(N_{H} \times N_{D}=5 \times 10^{6}\right)$. The result is a capacity of 0.92 Gbytes. The surface density (in the plane $D \times L$ ) is equal to $7.3 \mathrm{bits} / \mathrm{cm}^{2}$. Volume holographic storage can reach surface capacity of hundreds of bits per square micrometer by superimposing holograms in the same volume. Here the method we present is based on spatial multiplexing, i.e., it consists of nonoverlapping holograms. Such a restriction reduces the memory capacity, but offers the nice property of selective erasurerefreshing of data pages.

\section{Conclusion}

We have presented the SNR measurements of a novel selective read-write memory based on spatial multiplexing. The good SNR measurements obtained $(\simeq 4)$ are encouraging for the practical development of holographic memories. We presented a compact module using phase-conjugate reconstruction that allows greater memory capacity.

This research was supported by the Engineering Research Center for Neuromorphic Systems Engineering at the California Institute of Technology.

\section{References}

1. C. Moser, B. Schupp, and D. Psaltis, "Localized recording in doubly doped lithium niobate crystals," Opt. Lett. 25, 162-164 (2000).

2. C. Moser, I. Maravic, B. Schupp, A. Adibi, and D. Psaltis, "Diffraction efficiency of localized holograms in doubly doped $\mathrm{LiNbO}_{3}$ crystals," Opt. Lett. 25, 1243-1245 (2000).

3. D. A. Parthenopoulos and P. M. Rentzepis, "3-dimensional optical storage memory," Science 245, 843-845 (1989).

4. M. M. Wang, S. C. Esener, F. B. McCormick, I. Okgr, A. S. Dvornikov, and P. M. Rentzepis, "Experimental characterization of a two-photon memory," Opt. Lett. 22, 558-560 (1997).

5. A. Adibi, K. Buse, and D. Psaltis, "Sensitivity improvement in two-center holographic recording," Opt. Lett. 25, 539-541 (2000).

6. K. Buse, A. Adibi, and D. Psaltis, "Non-volatile holographic storage in doubly doped lithium niobate crystals," Nature (London) 393, 665-667 (1998).

7. M. C. Bashaw, A. Aharoni, and L. Hesselink, "Phase-conjugate replay for $a$-axis strontium barium niobate single-crystal fibers," Opt. Lett. 18, 2059-2061 (1993).

8. F. Ito and K.-I. Kitayama, "Holographic image storage in $\mathrm{LiNbO}_{3}$ fibers with compensation for intrasignal photorefractive coupling," J. Opt. Soc. Am. B. 9, 1432-1439 (1992). 